\title{
Effects of Electrolyte Beverage on Preventing Dehydration Among Workers in Different Enviromental Temperature
}

\author{
Ermita I.Ibrahim Ilyas, ${ }^{1}$ Saptawati Bardosono, ${ }^{2}$ Juwalita Surapsari, ${ }^{2}$ Hans-Joachim Freisleben ${ }^{3}$ \\ Received $18^{\text {th }}$ December 2017, \\ Accepted $22^{\text {th }}$ January 2018 \\ 1. Department of Medical Physiology, Faculty of Medicine, Universitas Indonesia, Jakarta, Indonesia \\ 2. Department of Nutrition Medical Faculty Universitas Indonesia-Cipto Mangunkusumo General \\ Hospital \\ Link to DOI: \\ 10.25220/WNJ/V01.i2.0007 \\ 3. Medical Research Unit, Medical Faculty, Universitas Indonesia
}

Journal Website:

www.worldnutrijournal.org

\begin{abstract}
Introduction: Water and electrolyte balance is important to maintain cognitive and physical performance, especially in hot environment. This study aimed to evaluate the effects of two different type of fluid intake at the workplace in preventing dehydration among male workers working in a hot and conveniently cool environment.

Methods: This randomized double-blinded placebo controlled trial study was performed in two appointed factories in West-Java in January-February 2012. Seventy-eight healthy male subjects, age 25-45 years old were selected and they were grouped based on their working environmental temperature, i.e. hot and conveniently cool environment. The subjects were randomly allocated by using crossover approach, to have non-electrolyte beverage (plain water) and electrolyte drink in the workplace for 2 days, respectively. Hydration and electrolyte biomarkers were collected from blood and urine samples at before and after the intervention.

Results: At baseline, subjects of the hot environment workplace had higher daily working hours, hemoglobin, hematocrit, blood viscosity, and blood sodium concentration as compared to those of conveniently cool environment $(p<0.05)$. After the intervention, for the subjects in hot environment alone, there were significantly lower value of blood viscosity, hemoglobin, and hematocrit, but significantly higher value for blood sodium, USG (urine specific gravity), pH, urinary sodium, urinary potassium and urinary chloride $(p<0.05)$, among subjects who had the electrolyte drink compared to the plain water.

Conclusion: This study confirmed that consumption of electrolyte beverage during working in hot environment temperature could help to improve hydration status and electrolyte concentration.

Keywords hydration, electrolyte beverage, hot environment, workers
\end{abstract}

\section{Introduction}

Dehydration is a condition of body water deficit. In

Corresponding author:

Ermita I. Ibrahim Ilyas

Department of Medical Physiology Medical Faculty

of Medicine, Universitas Indonesia

Email address : ermitailyas@gmail.com general, according to the research by The Indonesian Regional Hydration Study (THIRST) held on 2009, 46.1\% of population in Indonesia had mild dehydration. ${ }^{1}$ In a physical work setting, dehydration may result from the excessive sweat output compared to the water intake. Hydration in the work place became a special issue in which it can affect productivity, safety, and cost. 
Physical work increases heat production in the body which needs to be dissipated out from the body to achieve body heat balance. ${ }^{2}$ In a warm environment, excess of metabolic heat is dissipated to the environment by combination of conduction, convection, radiation, and evaporation of the sweat. When the environmental temperature approaches and exceeds the skin temperature, dry heat loss (by conduction, convection, and radiation) diminishes and is replaced by the 'heat gain'. In that condition, the only available heat loss mechanism is by sweat evaporation, and the sweat rate increases. ${ }^{3}$ Severity of sweat losses during work in a hot environment is dependent primarily on work intensity and duration. Sweating draws water from vascular, interstitial, and extra-cellular fluid compartments resulting in the secretion of hypotonic sweat. Metabolic heat production is balanced by both dry and evaporative (sweating) heat loss, but very high metabolic rates coupled with warm weather demands a larger thermal requirement for evaporative cooling, leading to greater sweat losses and subsequently larger water requirement.

It is widely known that water and electrolyte balance is very important to be continuously maintained to sustain cognitive and physical performance. Body water deficit results from hot weather and/or low water consumption, even as much as two percent of body weight, may impair physical and/or work performance. Any water deficit will affect performance in athlete or worker and lead to altered physical function and health. Therefore, an adequate level of hydration is essential. The possibility of water and electrolyte losses through sweating should be put into consideration for people working in hot environments. Electrolyte beverage usually contains water, electrolytes such as sodium, potassium, calcium, and carbohydrate as well. The addition of electrolytes to ingested water will keep the plasma electrolyte concentration and osmolality stable thus the water diuresis can be avoided. Electrolyte beverage has been reported to be more effective in retaining water in the body and preventing hemoconcentration than plain water. Ingestion of plain water will cause voluntary dehydration due to decrease of the osmolality that lead to stimulate the water diuresis. Chang et al. ${ }^{4}$ in 2010, reported that the recovery from high blood viscosity induced by dehydration was faster with electrolyte beverage consumption than with plain water or tea. A strong correlation between hematocrit and blood viscosity suggests that fluid retained in the body reduced the hemoconcentration and blood viscosity. ${ }^{4}$ In our preliminary study showed that workers in two factories, with different environment which are hot and conveniently cool environment were prone to dehydration. We found that hemoglobin concentration, hematocrit, blood viscosity and blood sodium concentration of workers in hot environment were significantly higher than workers in conveniently cool environment. ${ }^{5}$

This present study was performed to evaluate the effect of electrolyte beverage in preventing dehydration from workers, especially noted from clinical symptoms and laboratory measurements (hemoglobin, hematocrit, blood viscosity, blood and urine minerals, urine specific gravity and $\mathrm{pH}$ ) and to compare the effects of electrolyte beverage and plain water in preventing dehydration in workers in real work setting. It is very important to prevent worker from dehydration and to protect or reduce the risk of having cardiovascular problem which can be caused by hemoconcentration and high blood viscosity. This study hopefully could give information needed in the occupational field settings with workers who work in a hot and conveniently cool environment, who are considered to be at risk of dehydration that might lower their productivity. Therefore, the management departments may obtain some insights from this research to overcome this problem.

\section{Methods:}

\section{Study Design}

The study was a crossover, randomized, doubleblinded placebo controlled trial involving apparently healthy male subjects workers age 2545 years old, working minimally for 8 hours perday in two selected manufactures in Cibitung, West-Java. They did not have renal disease and diabetes mellitus, and willing to participate in the study by signing the informed consent.

We could not find any references on the difference effect of electrolyte drink versus the 
drinking water on hydration status and electrolytes concentration. Therefore this study was conducted as a preliminary study to have 40 subjects for each environment condition or minimal 80 subjects for the total sample.

After signing the informed consent, the selected subjects were classified according to their environmental temperature, i.e. the hot and conveniently cool environments, which were determined by room temperature measurement (36- $38^{\circ} \mathrm{C}$ versus $20-22{ }^{\circ} \mathrm{C}$, respectively). Subjects working in hot environment were those working close to the heat. Subjects working in conveniently cool environment were those doing the administration task in the office facilitated with air conditioner. The subjects of each environment temperature were randomly allocated into two different interventions by using crossover approach, in which each subject acted as his own control (Figure 1). Then, each subject will get both interventions for two days period. Subjects were advised to consume $300 \mathrm{~mL}$ of the provided fluid every 30 minutes for those working in hot environment and every hour for those working in the conveniently cool environment. Each subject had two days of non-electrolyte beverage (plain water) and, after the crossover, had two days of electrolyte beverage as well. These drinks were provided during 8-hours working period in 4 days of intervention (the subjects can have ad libitum drink), served personally in similar shape and color glasses. The total fluid intake was recorded through measuring the left over drink. During the intervention, lunch and break time snacks were provided with calorie contributing to $30-40 \%$ of the total calorie.

\section{Study overview}

This study was done at two automobile spare-parts factories in Cibitung West Java in January to February 2012. Before starting the recruitment and including the subjects into the study, informed consent was asked and recorded. This study received ethical approval from the Ethics Committee of the Faculty of Medicine Universitas Indonesia (No. 30/PT02/FK/ETIK/2012, January 18, 2012). All subjects signed the informed consent form to show their willingness to participate in the study.

\section{Data Collection}

Subjects were interviewed regarding their sociodemographic characteristic, employment duration and medical history. Interview was needed to clarify the food record as well. Food record method was used to assess energy intake and was done in 4 consecutive days during the intervention period. Anthropometric measurements were done before the intervention, which included body weight and height to calculate the body mass index. Vital sign measurements including blood pressure and heart rates were collected through physical examination.

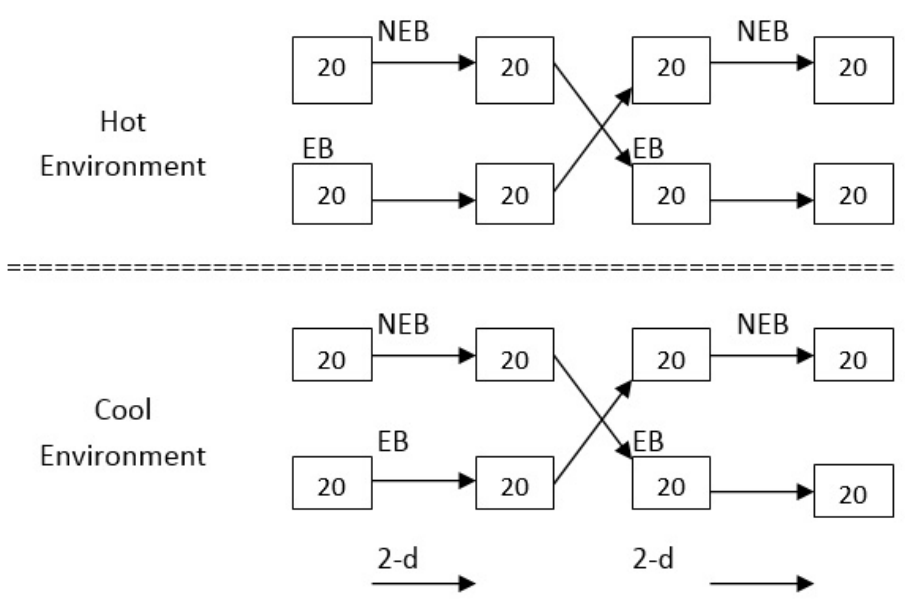

Figure 1

Diagram of study design to see the effect of electrolyte beverage in preventing dehydration among workers in two different environments (EB, electrolyte drink; NEB non-electrolyte drink or plain water) 
Blood and urine samples were collected before intervention to obtain baseline data on hemoglobin, hematocrit, blood viscosity, blood glucose, renal function (estimated creatinine-clearance test), blood and urine electrolytes (sodium, potassium and chloride), urine color, $\mathrm{pH}$, and urine specific gravity (USG) by using standardized procedures. The data was collected again after two days after the working time during the four days study period to obtain data on the effect of each of the intervention.

\section{Statistical Analyses}

Data was recorded using a special form and was edited, coded, and administered into the working sheet by using statistical program for social sciences (SPSS) software version 20. All data was presented accordingly based on its normality distribution, and analyzed by using unpaired-T test or Mann-Whitney test, paired-T test or Wilcoxon test, and Chi-square or McNemar test.

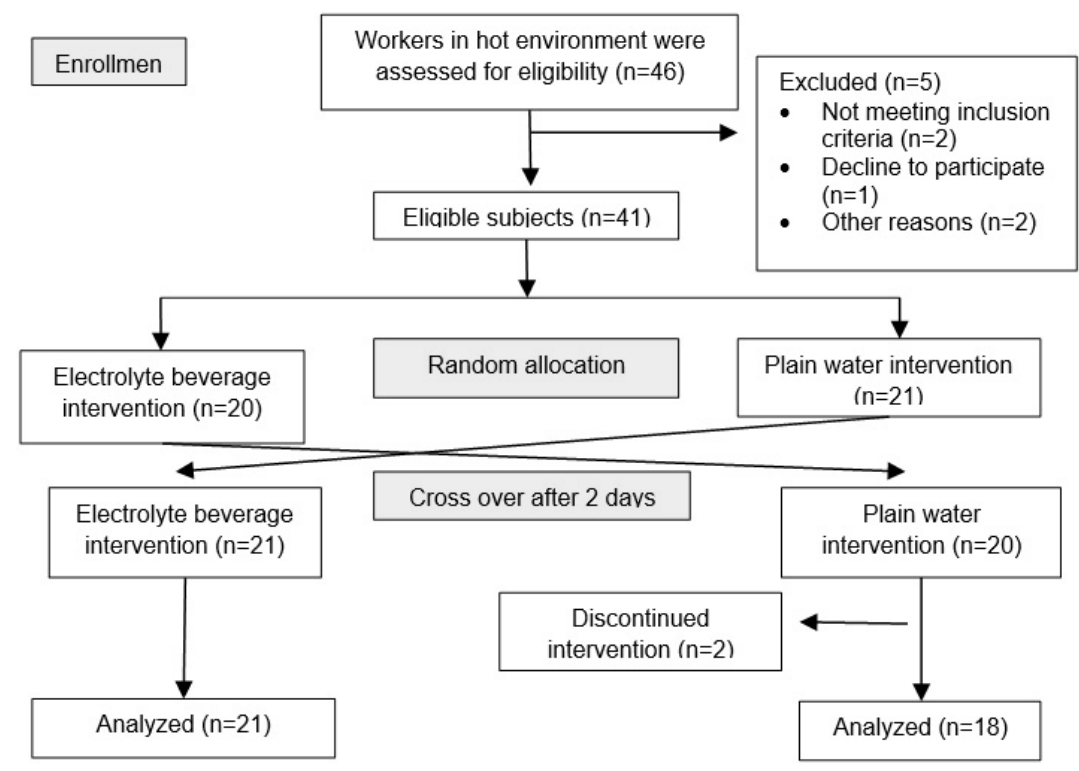

Figure 2A

Flow diagram of subjects in hot environment workplace

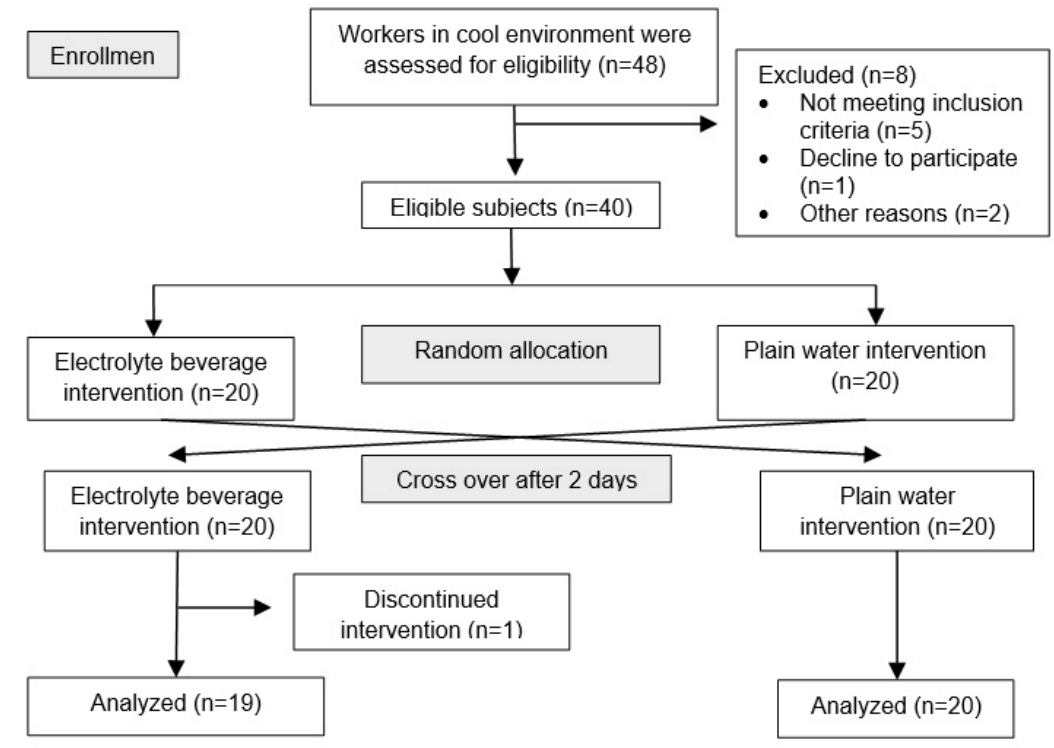

Figure 2B

Flow diagram of subjects in conveniently cool environment workplace 


\section{Results}

The recruitment of the subjects started from $6^{\text {th }}$ January 2012 and ended on $3^{\text {rd }}$ February 2012. Figure 2A and Figure 2B show the flow of the intervention and data collection scheme, and we analyzed 39 subjects receiving both interventions in each workplace conditions, i.e. hot and conveniently cool environment.

Before the intervention, this study found that there were significant differences in several hydration and electrolytes biomarkers, in which hemoglobin, hematocrit, blood viscosity and blood sodium were higher among those working in hot environment compared to those working in the conveniently cool environment workplace $(\mathrm{P}<0.05)$, as shown in Table 1. The general conditions of the subjects in both temperature workplaces were not affected by the interventions, as presented in Table 2.
In the blood measurement, there were significant lower values of blood viscosity, hemoglobin, hematocrit, and blood sodium concentration among subjects after receiving electrolyte drink compared to subjects receiving plain water for two days, but only among those working in hot environment workplace, as shown in Table 3.

However, Table 4 shows that those having electrolyte drink had significantly higher USG, $\mathrm{pH}$, urinary sodium, potassium and chloride compared to those having plain water in which, again, only found among those working in hot environment workplace.

Table 1 General characteristics of the subjects by different workplace environment before interventions

\begin{tabular}{|c|c|c|c|}
\hline Variables & Hot environment & Cool environment & P-value \\
\hline Age, $y$ & $29(25-44)^{\wedge}$ & $30(25-45)^{\wedge}$ & $0.086^{*}$ \\
\hline Duration of working, $y$ & $8(1-22)^{\wedge}$ & $8(1-30)^{\wedge}$ & $0.695 *$ \\
\hline Working hours/day & $12(8-12)^{\wedge}$ & $8(7-12)^{\wedge}$ & $<0.001 *$ \\
\hline Body weight, kg & $64.81 \pm 11.9^{\sim}$ & $68.97 \pm 12.0^{\sim}$ & $0.129 * *$ \\
\hline Body height, $\mathrm{m}$ & $1.67(1.55-1.87)^{\wedge}$ & $1.66(1.57-1.83)^{\wedge}$ & $0.682 *$ \\
\hline BMI, $\mathrm{kg} / \mathrm{m}^{2}$ & $23.6 \pm 4.8^{\sim}$ & $24.8 \pm 4.2^{\sim}$ & $0.227 * *$ \\
\hline Hemoglobin, g/dL & $15.6(12.3-18.0)^{\wedge}$ & $14.8(12.6-17.2)^{\wedge}$ & $0.017 *$ \\
\hline Hematocrit, \% & $46(39-49)^{\wedge}$ & $44(40-49)^{\wedge}$ & $0.040 *$ \\
\hline $\begin{array}{l}\text { Blood viscosity, } \\
\text { mPa.s }\end{array}$ & $23.0 \pm 8.2^{\sim}$ & $12.0 \pm 2.2^{\sim}$ & $<0.001 * *$ \\
\hline $\begin{array}{l}\text { Blood sodium, } \\
\mathrm{mOsm} / \mathrm{L}\end{array}$ & $140(136-145)^{\wedge}$ & $138(135-141)^{\wedge}$ & $<0.001 *$ \\
\hline USG & $1.0178 \pm 0.0076^{\sim}$ & $1.0187 \pm 0.0077^{\sim}$ & $0.626^{* *}$ \\
\hline Blood pressure, $\mathrm{mmHg}$ : & & & \\
\hline Systolic & $110(90-160)^{\wedge}$ & $120(80-150)^{\wedge}$ & $0.243 *$ \\
\hline Diastolic & $80(60-100)^{\wedge}$ & $80(60-100)^{\wedge}$ & $0.949 *$ \\
\hline
\end{tabular}

BMI, body mass index; USG, urine specific ${ }^{\wedge}$ median (minimum-maximum); $\sim$ mean (SD),

*Mann-Whitney test; **unpaired-t test 
Table 2 General conditions of the subjects by beverage-drink type intake in different workplace environments

\begin{tabular}{|c|c|c|c|c|c|c|}
\hline \multirow[t]{2}{*}{ General Conditions } & \multicolumn{3}{|c|}{ Hot-environment } & \multicolumn{3}{|c|}{ Cool-environment } \\
\hline & Plain water & Electrolyte drink & P-value & Plain water & $\begin{array}{l}\text { Electrolyte } \\
\text { drink }\end{array}$ & P-value \\
\hline \multicolumn{7}{|l|}{ Systolic BP (mmHg) } \\
\hline \multirow[t]{2}{*}{ Baseline } & 120 & 110 & $0.907 *$ & 110 & 120 & $0.817 *$ \\
\hline & $(100-140)^{\wedge}$ & $(90-160)^{\wedge}$ & & $(80-140)^{\wedge}$ & $(90-150)^{\wedge}$ & \\
\hline \multirow[t]{2}{*}{ After two days } & 120 & 120 & $0.683 *$ & 110 & 120 & $0.161^{*}$ \\
\hline & $(100-150)^{\wedge}$ & $(100-150)^{\wedge}$ & & $(100-150)^{\wedge}$ & $(100-150)^{\wedge}$ & \\
\hline \multicolumn{7}{|l|}{ Diastolic BP $(\mathrm{mmHg})$ : } \\
\hline \multirow[t]{2}{*}{ Baseline } & 80 & 80 & $0.861 *$ & 70 & 80 & $0.238^{*}$ \\
\hline & $(60-90)^{\wedge}$ & $(60-100)^{\wedge}$ & & $(60-100)^{\wedge}$ & $(60-100)^{\wedge}$ & \\
\hline \multirow[t]{2}{*}{ After two days } & 80 & 80 & $0.397 *$ & 80 & 80 & $0.285^{*}$ \\
\hline & $(70-100)^{\wedge}$ & $(60-90)^{\wedge}$ & & $(60-100)^{\wedge}$ & $(60-100)^{\wedge}$ & \\
\hline \multicolumn{7}{|l|}{ Heart rate (times/minute): } \\
\hline Baseline & $72(60-88)^{\wedge}$ & $72 \pm 6 \sim$ & $0.900 *$ & $78 \pm 7 \sim$ & $72 \pm 7 \sim$ & $0.706^{* *}$ \\
\hline After two days & $78(60-84)^{\wedge}$ & $78(66-84)^{\wedge}$ & $0.408^{*}$ & $73 \pm 8 \sim$ & $74 \pm 8 \sim$ & $0.498 * *$ \\
\hline \multicolumn{7}{|l|}{ Fluid intake $(\mathrm{mL})$ : } \\
\hline Baseline & $3732.8 \pm 745.9 \sim$ & $3640.0 \pm 666.4 \sim$ & $0.219^{* *}$ & $1785.5 \pm 489.0 \sim$ & $1805.3 \pm 459.7 \sim$ & $0.781 * *$ \\
\hline \multirow[t]{2}{*}{ After two days } & $3813.6 \pm 6653.4 \sim$ & 4059 & $0.159^{*}$ & $1749 \pm 518.0 \sim$ & $1778 \pm 492.9 \sim$ & $0.698^{* *}$ \\
\hline & & $(1660-4800)^{\wedge}$ & & & & \\
\hline
\end{tabular}

${ }^{\wedge}$ median (minimum-maximum); mean (SD), *Wilcoxon test; ${ }^{* *}$ paired-t test 
Table 3 Hydration biomarkers of the subjects taken from blood sample by beverage-drink type intake in different workplace environments

\begin{tabular}{|c|c|c|c|c|c|c|}
\hline \multirow{2}{*}{ Hydration biomarkers } & \multicolumn{3}{|c|}{ Hot-environment } & \multicolumn{3}{|c|}{ Cool-environment } \\
\hline & Plain water & $\begin{array}{l}\text { Electrolyte } \\
\text { drink }\end{array}$ & P-value & Plain water & $\begin{array}{l}\text { Electrolyte } \\
\text { drink }\end{array}$ & P-value \\
\hline Baseline & $\begin{array}{c}16.6 \\
(8.3-36.9)^{\wedge}\end{array}$ & $\begin{array}{c}15.0 \\
(7.4-39.2)^{\wedge}\end{array}$ & $0.685^{*}$ & $12.1 \pm 2.3 \sim$ & $12.1 \pm 2.0 \sim$ & $0.939^{* *}$ \\
\hline Changes after - baseline & $\begin{array}{c}-2.0 \\
(-16.0-3.1)^{\wedge}\end{array}$ & $\begin{array}{c}-4.1 \\
(-22.1-1.1)^{\wedge}\end{array}$ & $0.277^{*}$ & $-0.5 \pm 2.4 \sim$ & $0.05 \pm 2.3 \sim$ & $0.371^{* *}$ \\
\hline \multicolumn{7}{|l|}{ Hemoglobin (mg/dL): } \\
\hline Changes after - baseline & $-0.1 \pm 0.8 \sim$ & $\begin{array}{c}-0.4 \\
(-2.0-0.8)^{\wedge}\end{array}$ & $0.088^{*}$ & $-0.2 \pm 0.5 \sim$ & $-0.1 \pm 0.6 \sim$ & $0.295^{* *}$ \\
\hline
\end{tabular}


Table 3 (continued)

Hydration biomarkers

\begin{tabular}{lccccc} 
& Hot-environment & \multicolumn{3}{c}{ Cool-environment } \\
\hline Plain water & $\begin{array}{c}\text { Electrolyte } \\
\text { drink }\end{array}$ & P-value & Plain water & $\begin{array}{c}\text { Electrolyte } \\
\text { drink }\end{array}$ & P-value \\
& & &
\end{tabular}

Hematocrit (\%):

Baseline

After two days

Changes after - baseline

Sodium $(\mathrm{mOsm} / \mathrm{L})$ :

Baseline

After two days

Changes after - baseline

Potassium (mOsm/L):

Baseline

After two days

Changes after - baseline
$44.5 \pm 2.8 \sim$

45.0

$0.571^{*}$

$44(39-48)^{\wedge}$

$44(40-49)^{\wedge}$

$0.958^{*}$

$(38-49)^{\wedge}$

$44.3 \pm 2.6 \sim \quad 43.7 \pm 2.2 \sim$

\subsection{9**}

$43(41-48)^{\wedge}$

$44(39-50)^{\wedge}$

$0.167^{*}$

$-0.2 \pm 2.3 \sim$

$-1.0(-5-3.0)^{\wedge}$

$0.118 *$

$0(-4-3.0)^{\wedge}$

$-0.1 \pm 2.0 \sim$

$0.478 *$

$141.2 \pm 1.9 \sim$

141.0

$0.663 *$

139

138

$0.078 *$

$(136-145)^{\wedge}$

141.0

141.0

$0.024 *$

$(137-141)^{\wedge}$

$(135-141)^{\wedge}$

139

139

$0.114^{*}$

$(136-143)^{\wedge}$

$(138-146)^{\wedge}$

$-1.0 \quad 0(-3.0-6.0)^{\wedge}$

$(136-140)^{\wedge}$

$(137-141)^{\wedge}$

$90 *$

$0(-3.0-3.0)^{\wedge}$

$0(-3.0-6.0)^{\wedge}$

$0.051 *$

$(-4.0-3.0)^{\wedge}$

$\begin{array}{cccccc}4.0(3.4-5.0)^{\wedge} & 4.0(3.4-5.0)^{\wedge} & 0.468^{*} & 4.0(3.6-6.0)^{\wedge} & 4.0(3.5-5.0)^{\wedge} & 0.496^{*} \\ 4.0(3.2-4.4)^{\wedge} & 4.0(3.4-5.0)^{\wedge} & 0.671^{*} & 3.8(3.4-4.4)^{\wedge} & 3.9(3.3-4.5)^{\wedge} & 0.423^{*} \\ 0(-1.6-0.4)^{\wedge} & 0(-1.0-1.0)^{\wedge} & 0.396^{*} & -0.3 & -0.3 & 0.209^{*}\end{array}$


Table 3 (continued)

\begin{tabular}{|c|c|c|c|c|c|c|}
\hline \multirow[t]{2}{*}{ Hydration biomarkers } & \multicolumn{3}{|c|}{ Hot-environment } & \multicolumn{3}{|c|}{ Cool-environment } \\
\hline & Plain water & $\begin{array}{l}\text { Electrolyte } \\
\text { drink }\end{array}$ & P-value & Plain water & $\begin{array}{l}\text { Electrolyte } \\
\text { drink }\end{array}$ & P-value \\
\hline \multicolumn{7}{|l|}{ Chloride $(\mathrm{mOsm} / \mathrm{L})$ : } \\
\hline \multirow[t]{2}{*}{ Baseline } & 101.0 & 101.0 & $0.937^{*}$ & 102 & 102 & $0.342 *$ \\
\hline & $(97-105)^{\wedge}$ & $(98-104)^{\wedge}$ & & $(100-107)^{\wedge}$ & $(99-106)^{\wedge}$ & \\
\hline \multirow[t]{2}{*}{ After two days } & 100.0 & 101.0 & $0.185^{*}$ & 102 & 102 & $0.483 *$ \\
\hline & $(97-105)^{\wedge}$ & $(97-105)^{\wedge}$ & & $(100-105)^{\wedge}$ & $(100-106)^{\wedge}$ & \\
\hline Changes after - baseline & $-0.2 \pm 1.8 \sim$ & $0(-4.0-4.0)^{\wedge}$ & $0.686^{*}$ & $0(-4.0-3.0) \sim$ & $0(-3.0-3.0) \sim$ & $0.977 *$ \\
\hline
\end{tabular}

${ }^{\wedge}$ median (minimum-maximum); $\sim$ mean $(\mathrm{SD}), *$ Wilcoxon test; $* *$ paired-t test

Table 4 Hydration biomarkers of the subjects taken from urine sample by beverage-drink type intake in different workplace environments

\begin{tabular}{|c|c|c|c|c|c|c|}
\hline \multirow{2}{*}{$\begin{array}{l}\text { Hydration biomarkers } \\
\text { (from urine-sample) }\end{array}$} & \multicolumn{3}{|c|}{ Hot-environment } & \multicolumn{3}{|c|}{ Cool-environment } \\
\hline & Plain water & $\begin{array}{l}\text { Electrolyte } \\
\text { drink }\end{array}$ & P-value & Plain water & $\begin{array}{l}\text { Electrolyte } \\
\text { drink }\end{array}$ & P-value \\
\hline \multicolumn{7}{|l|}{ USG status: } \\
\hline \multirow[t]{2}{*}{ Baseline } & 1.009 & $1.014 \pm 0.009 \sim$ & $0.451 *$ & 1.009 & $1.015 \pm 0.007 \sim$ & $0.207 *$ \\
\hline & $(1.001-1.033)^{\wedge}$ & & & $(1.003-1.032)^{\wedge}$ & & \\
\hline \multirow[t]{2}{*}{ After two days } & 1.004 & 1.006 & $<0.001 *$ & 1.006 & 1.007 & $0.401 *$ \\
\hline & $(1.001-1.022)^{\wedge}$ & $(1.001-1.032)^{\wedge}$ & & $(1.002-1.029)^{\wedge}$ & $(1.003-1.026)^{\wedge}$ & \\
\hline \multirow[t]{2}{*}{ Changes after - baseline } & -0.007 & -0.003 & $0.115^{* *}$ & -0.005 & -0.007 & $0.454 * *$ \\
\hline & $\pm 0.010 \sim$ & $\pm 0.010 \sim$ & & $\pm 0.010 \sim$ & $\pm 0.008 \sim$ & \\
\hline
\end{tabular}


Table 4 (continued)

\begin{tabular}{|c|c|c|c|c|c|c|}
\hline \multirow{2}{*}{$\begin{array}{l}\text { Hydration biomarkers } \\
\text { (from urine-sample) }\end{array}$} & \multicolumn{3}{|c|}{ Hot-environment } & \multicolumn{3}{|c|}{ Cool-environment } \\
\hline & Plain water & $\begin{array}{l}\text { Electrolyte } \\
\text { drink }\end{array}$ & P-value & Plain water & $\begin{array}{l}\text { Electrolyte } \\
\text { drink }\end{array}$ & P-value \\
\hline \multicolumn{7}{|l|}{ pH: } \\
\hline Baseline & $6(5-7)^{\wedge}$ & $6(5-7)^{\wedge}$ & $0.123^{*}$ & $6(5-7)^{\wedge}$ & $6.5(5-8)^{\wedge}$ & $0.664 *$ \\
\hline After two days & $6(5-7)^{\wedge}$ & $6(5-8)^{\wedge}$ & $0.003 *$ & $6(5-7)^{\wedge}$ & $6(5-7)^{\wedge}$ & $0.293 *$ \\
\hline Changes after - baseline & $0(-1.0-1.0)^{\wedge}$ & $0(-1.0-2.0)^{\wedge}$ & $0.003 *$ & $0(-2.0-2.0)^{\wedge}$ & $0.2 \pm 0.8 \sim$ & $0.947 *$ \\
\hline \multicolumn{7}{|l|}{ Normal urine color, $\mathrm{n}(\%)$ : } \\
\hline Baseline & $32(82.1)$ & $35(89.7)$ & $0.549 * * *$ & $35(89.7)$ & $33(84.6)$ & $0.727 * * *$ \\
\hline After two days & $39(100)$ & $36(92.3)$ & - & $39(100)$ & $39(100)$ & - \\
\hline \multicolumn{7}{|l|}{ Sodium $(\mathrm{mOsm} / \mathrm{L})$ : } \\
\hline \multirow[t]{2}{*}{ Baseline } & 64 & 95 & $0.553^{*}$ & 70.8 & 94.0 & $0.379 *$ \\
\hline & $(14-232)^{\wedge}$ & $(10-256)^{\wedge}$ & & $(0-299.0)^{\wedge}$ & $(30.0-266.3)^{\wedge}$ & \\
\hline \multirow[t]{2}{*}{ After two days } & 29.8 & $62(13-301)^{\wedge}$ & $<0.001 *$ & 41.0 & 50.4 & $0.426^{*}$ \\
\hline & $(13.0-143.8)^{\wedge}$ & & & $(13.1-180.6)^{\wedge}$ & $(15.3-194.1)^{\wedge}$ & \\
\hline \multirow{2}{*}{ Changes after - baseline } & -41.0 & $3.2 \pm 94.4 \sim$ & $0.054^{*}$ & -13.7 & -36.4 & $0.577 *$ \\
\hline & $(-192.0-104.7)^{\wedge}$ & & & $(-281.5-103.6)^{\wedge}$ & $(-244.5-97.4)^{\wedge}$ & \\
\hline
\end{tabular}


Table 4 (continued)

\begin{tabular}{|c|c|c|c|c|c|c|}
\hline \multirow{2}{*}{$\begin{array}{l}\text { Hydration biomarkers } \\
\text { (from urine-sample) }\end{array}$} & \multicolumn{3}{|c|}{ Hot-environment } & \multicolumn{3}{|c|}{ Cool-environment } \\
\hline & Plain water & $\begin{array}{l}\text { Electrolyte } \\
\text { drink }\end{array}$ & P-value & Plain water & $\begin{array}{l}\text { Electrolyte } \\
\text { drink }\end{array}$ & P-value \\
\hline \multicolumn{7}{|l|}{ Potassium $(\mathrm{mOsm} / \mathrm{L})$ : } \\
\hline Baseline & $\begin{array}{c}16.0 \\
(1.0-97.2)^{\wedge}\end{array}$ & $\begin{array}{c}20.0 \\
(2.0-93.0)^{\wedge}\end{array}$ & $0.942 *$ & $\begin{array}{c}15.0 \\
(0-110.5)^{\wedge}\end{array}$ & $\begin{array}{c}23.7 \\
(4.4-109.0)^{\wedge}\end{array}$ & $0.302 *$ \\
\hline After two days & $\begin{array}{c}6.0 \\
(1.0-51.2)^{\wedge}\end{array}$ & $\begin{array}{c}11.0 \\
(1.0-94.5)^{\wedge}\end{array}$ & $<0.001 *$ & $\begin{array}{c}8.5 \\
(2.5-46.0)^{\wedge}\end{array}$ & $\begin{array}{c}9.4 \\
(3.2-73.3)^{\wedge}\end{array}$ & $0.384^{*}$ \\
\hline Changes after - baseline & $\begin{array}{c}-7.0 \\
(-71.0-18.0)^{\wedge}\end{array}$ & $\begin{array}{c}-1.0 \\
(-92.0-74.5)^{\wedge}\end{array}$ & $0.246^{*}$ & $\begin{array}{c}-2.8 \\
(-79.4-20.0)^{\wedge}\end{array}$ & $\begin{array}{c}-13.2 \\
(-86.4-11.8)^{\wedge}\end{array}$ & $0.468^{*}$ \\
\hline \multicolumn{7}{|l|}{ Chloride $(\mathrm{mOsm} / \mathrm{L})$ : } \\
\hline Baseline & $\begin{array}{c}65.0 \\
(7.1-356.0)^{\wedge}\end{array}$ & $116.4 \pm 84.8 \sim$ & $0.577^{*}$ & $\begin{array}{c}27.6 \\
(0-199.1)^{\wedge}\end{array}$ & $122.3 \pm 75.4 \sim$ & $<0.001 *$ \\
\hline After two days & $\begin{array}{c}23.0 \\
(7.0-146.7)^{\wedge}\end{array}$ & $\begin{array}{c}100.0 \\
(10.0-274.3)^{\wedge}\end{array}$ & $<0.001 *$ & $\begin{array}{c}35.2 \\
(8.5-167.3)^{\wedge}\end{array}$ & $\begin{array}{c}45.4 \\
(10.5-177.7)^{\wedge}\end{array}$ & $0.276^{*}$ \\
\hline Changes after - baseline & $\begin{array}{c}-31.0 \\
(-283.0-118.5)^{\wedge}\end{array}$ & $-25.7 \pm 114.5 \sim$ & $0.161 *$ & $\begin{array}{c}5.4 \\
(-78.4-128.5)^{\wedge}\end{array}$ & $\begin{array}{c}-41.7 \\
(-247.2-94.6)^{\wedge}\end{array}$ & $<0.001 *$ \\
\hline
\end{tabular}

${ }^{\wedge}$ median (minimum-maximum); $\sim$ mean (SD),

*Wilcoxon test; **paired-t test; ***McNemar-test

$48 \mid$ World.Nutr.J 


\section{Discussion}

This study aimed to evaluate the effect of electrolyte beverage in preventing dehydration for workers, especially noted from clinical symptoms and laboratory measurements (hemoglobin, hematocrit, blood viscosity, blood and urine minerals, urine specific gravity and $\mathrm{pH}$ ). This study's objective was also to compare the effect of electrolyte beverage and plain water (nonelectrolyte drink) in promoting hydration and electrolytes balance among the workers in hot and conveniently cool environment. We tried to keep the subjects as stated in the protocol of the study, however, two subjects from the hot environment and one subject from the cool environment had to leave due to urgent reasonable reasons (Figure 2A and Figure 2B).

Based on work environment difference, the subjects had similar age, however they had different working hours (Table 1). It was shown that subjects working in the hot environment had significantly longer working hours compared to those working in the cool environment. By working in a hot environment for a longer period, the risk to become dehydrated is higher unless the workers are used to drinking sufficiently. If we could extrapolate by using an example of very active fire fighters, then they should have daily water requirements of about $7 \mathrm{~L} /$ day. ${ }^{6}$

Based on the blood analyses, Table 1 shows several indices of hydration status. There were significantly higher hemoglobin concentration, hematocrit, blood viscosity and blood sodium among subjects working in hot environment compared to the subject working in the cool environment. Particularly, $79.5 \%$ of subjects working in hot environment had high blood viscosity compared to subjects in the cool environment $(25.6 \%)$. These showed that workers who work in the hot environment are at higher risk of falling into a dehydration state compared to those working in the conveniently cool environment. The sweating process involves the fluid loss from the extracellular compartment including fluid from the vascular. Hematocrit can be described as relationship between the cellular volume compared to total blood volume. Its level increases when the total RBC amount increases or when a person losses fluid which leads to a decrease of plasma volume, which happens in sweating process. Working in a hot environment increases sweat rates which results in decrease of plasma volume. The blood viscosity increases along with the increasing hematocrit. ${ }^{7}$

The most widely investigated are body mass changes, blood indices, urine indices and bioelectrical impedance analysis. ${ }^{8}$ Measurement of haemoglobin concentration and hematocrit has the potential to be used as a marker or change in hydration status. However, Armstrong et $\mathrm{al}^{9}$ (1994) stated that hematologic measurements are not as sensitive to mild hypohydration as the certain urinary indices. This perhaps suggests that plasma volume is defended in an attempt to maintain cardiovascular stability, and so plasma variables will not be affected by hypohydration or dehydration until a certain degree of body water loss has occurred.

The subjects working in hot environment had higher sodium level than those working in cool environment. This higher levels of sodium is probably caused by two things: first, the water loss from the skin from sweating process made extracellular fluid depletion more excessive in the subjects working in hot environment. Second, the lower water intake of the subjects in hot environment might be the cause of this higher sodium level, which is shown at Tabel 2. As a primary cation in extracellular fluid (ECF), any loss of water will increase sodium concentration in ECF compartment, which in turn will increase plasma osmolality. Plasma osmolality also provides as a marker of dehydration level because it is closely controlled by homeostatic system, thus serving as a primary physiological signal to regulate water balance, i.e. changes in urine output and fluid intake. ${ }^{10-11}$

Plasma or serum sodium concentration and osmolality will increase when the water loss inducing dehydration is hypotonic with respect to plasma. An increase in these concentrations would be expected, as in many cases of hypohydration, including water loss by sweat secretion, urine production or diarrhea. Similar finding was reported by Armstrong et $\mathrm{al}^{9}$ (1994), which perhaps suggests that plasma volume is defended in an attempt to maintain cardiovascular stability. Thus, 
plasma variables will not be affected by dehydration until a certain degree of body water loss has occurred.

Baseline fluid intake (plain water and electrolyte beverage) in both groups, as seen in Table 2, are not significantly different. However, subjects working in hot environment had higher daily fluid requirements compared to subjects in cool environment, because the skin water loss is higher as well and this condition will increase the feeling of thirst. The lower water intake of subjects in hot environment is probably related to water provision. The water supply for workers in hot environment was only provided in a rest area which probably cannot be easily accessed by workers because they could not leave their work at any time and they were not provided with a water container at their work place. On the days of intervention, the median of water intake reached up to 3607-4142 $\mathrm{mL}$. During the interventions, the fluid intake was increase because the researchers provided the water in tumblers which were held in a carrying bag which then easily accessed and drank by the workers. As expected, the amounts of fluid consumptions during the intervention period were significantly higher among workers in the hot environment compared to those working in the cool environment. By providing the fluid at the workplace and giving easy access to the fluid supply, the workers in the hot environment voluntarily increased their fluid consumption which then significantly increased their total fluid intake.

By providing different types of fluid drink, i.e. plain water and isotonic drink, this study showed perceptual factor in relation to voluntary drinking. As widely known, voluntary drinking of a beverage is affected by its palatability, which is determined by its color, flavor, odor, and temperature. $^{12-17}$ The sweet flavour of a drink is a major factor in its palatability. However, in reality, people's prefer flavor differ, which depends on various factors, including ethnicity and cultural backgrounds. In this study, it was revealed that there was no difference in the volume of fluid intake between the two different types of provided drink $(p>0.05)$ both in the hot and cool environment workers (Table 2).

As seen in Table 2, there was no significant change neither in systolic nor diastolic blood pressure during the intervention in both environment. Intervention with plain water and isotonic fluid both could maintain the blood presssure in a stable condition. Dehydration will typically lower blood pressure slightly due to lower blood volume but this happens only in extreme cases. Extreme overhydration (faster than can be processed and expelled from the body) can lead to a raised blood pressure. The body regulates hormones to keep the blood pressure basically stable except in extreme cases. ${ }^{18}$

Heart rate is a vital sign to provide clues to the presence of many medical conditions. Reflex changes in heart rate are one of the body's most basic mechanisms for maintaining proper perfusion to the brain and other tissues. ${ }^{19}$ Perfusion is the flow of blood through an organ. Low blood volume caused by bleeding or dehydration results in the heart beating faster as it attempts to maintain adequate blood pressure. Excitement, stress, and anxiety activate the nervous system, which may also speed the heart rate and raise blood pressure. Total body water by weight was found to be related to diastolic blood pressure, $r=-0.56, p=0.01 .^{18}$

Table 3 shows that blood viscosity after intervention is significantly decreased for subjects in hot environment because they were potentially dehydrated induced by the high sweat rates. The isotonic drink had a lower effect on blood viscosity than the plain water as the isotonic drink contains electrolytes which pertains the plasma volume and avoids water diuresis. Hemoglobin and hematocrit were different after the intervention on both groups. However, there was no significant difference between the workers that drink plain water and electrolyte beverage. The lower hematocrit level during intervention suggested more liquid entering the intravascular space which then lowered the hematocrit level, in accordance to the relationship between the cellular volume to the total blood volume. $^{7}$ These findings remarked that fluid replacement, either with isotonic drink or plain water, could avoid the potential hemoconcentration during work. While the average blood sodium level of subjects in hot environment during intervention period was significantly different between plain water and electrolyte beverage in hot environment workers, it is not the same in cool convenient environment. 
Both interventions lowered the urine specific gravity in hot environment setting as the fluids diluted the urine (Table 4). The administration of isotonic drink first will produce lower urine specific gravity because it retained more fluid in the body. While in the cool environment setting, both interventions lowered the urine specific gravity but there was no difference between the plain water and electrolyte beverage as there were no fluid losses in this setting. The administration of both fluids in subjects of hot environment setting would lower the renal sodium excretion on the first phase, then the isotonic drink on the second phase would made an adequate level in the blood thus excreting more sodium in the urine. In the conveniently cool setting both intervention lowered the renal sodium excretion but no difference between the both fluid. In that setting the isotonic drink gave no benefit physiologically. Plain water and isotonic beverage both could maintain the body water of the subjects in hot environment, as both could replace the increasing water loss from sweating process. In the cool environment, the baseline body water, which was lower than the counterpart, increased after the administration of both fluids, but their peak were more prominent after the administration of isotonic fluid.

In conclusion consuming electrolyte beverage would prevent male workers aged 25-45 years, especially those working in a hot environment from dehydration as compared to nonelectrolyte beverage.

\section{Conflict of Interest}

Saptawati Bardosono is a one of editors but was not involved in the review or decision process for this article.

\section{Open Access}

This article is distributed under the terms of the Creative Commons Attribution 4.0 International License (http://creativecommons.org/licenses/by/4.0/), which permits unrestricted use, distribution, and reproduction in any medium, provided you give appropriate credit to the original author(s) and the source, provide a link to the Creative Commons license, and indicate if changes were made.

\section{Acknowledgement}

This study was supported by PT Amerta Indah Otsuka Indonesia.

\section{References}

1. Hardinsyah, Briawan D, Effendi, YH, Dwiriani CM, Dewi M, Damayanti E, Aries M 2010, Kebiasaan minum dan dan status hidrasi pada remaja dan dewasa di dua wilayah ekologi berbeda, Tim THIRST (The Indonesian Regional Hydration Study), FEMA IPB, FKM UNAIR dan FKM UNHAS, Jakarta.

2. Sawka MN, Montain SJ, Latzka WA. Hydration effects on thermoregulation and performance in the heat. Comparative Biochemistry and Physiology Part A: Molecular \& Integrative Physiology 2001 Apr;128(4):679-90. [Google Scholar]

3. Miller VS, Bates GP. Hydration, hydration, hydration. Annals of occupational hygiene 2009;54(2):134-6. [Google Scholar]

4. Chang CQ, Chen YB, Chen ZM, Zhang LT. Effects of a carbohydrate-electrolyte beverage on blood viscosity after dehydration in healthy adults. Chinese medical journal 2010;123(22):3220-5. [Google Scholar]

5. Bardosono S, Ilyas E. Health, nutrition and hydration status of Indonesian workers: a preliminary study in two different environmental settings. Medical Journal of Indonesia 2014;23(2):112. [Google Scholar]

6. Ruby BC, Shriver TC, Zderic TW, Sharkey BJ, Burks C, Tysk S. Total energy expenditure during arduous wildfire suppression. Medicine and Science in Sports and Exercise 2002;34(6):1048-54. [Google Scholar]

7. Karsheva M, Dinkova P, Pentchev I, Ivanova T. Blood rheology-a key for blood circulation in human body. Journal of the University of Chemical Technology and Metallurgy 2009;44(1):50-4. [Google Scholar] 
8. Shirreffs SM. Markers of hydration status. European journal of clinical nutrition 2003 Dec;57. [Google Scholar]

9. Armstrong LE, Maresh CM, Castellani JW, Bergeron MF, Kenefick RW, LaGasse KE, et al. Urinary indices of hydration status. International journal of sport nutrition 1994;4(3):265-79. [Google Scholar]

10. Andreoli TE, Reeves WB, Bichet DG 2000, Endocrin control of water balance, in Fray JCS, Goodman HM (ed.), Handbook of Physiology: Endocrin regulation of water and electrolyte balance, Oxford University Press, New York, pp. 530-569.

11. Knepper MA, Valtin H, Sands JM 2000, Renal actions of vasopressin, in Fray JC, Goodman HM(ed.), Handbook of Physiology: Endocrin regulation of water and electrolyte balance, Oxford University Press, New York, pp. 496-529.

12. Boulze D, Montastruc P, Cabanac M. Water intake, pleasure and water temperature in humans. Physiology \& behavior 1983;30(1):97-102. [Google Scholar]

13. Hubbard RW, Sandick BL, Matthew WT, Francesconi RP, Sampson JB, Durkot MJ, et al. Voluntary dehydration and alliesthesia for water. Journal of Applied Physiology 1984;57(3):868-73. [Google Scholar]

14. Meyer F, Bar-Or O, Salsberg A, Passe D. Hypohydration during exercise in children: effect on thirst, drink preferences, and rehydration. International Journal of Sport Nutrition 1994;4(1):22-35. [Google Scholar]

15. Szlyk PC, Sils IV, Francesconi RP, Hubbard RW, Armstrong LE. Effects of water temperature and flavoring on voluntary dehydration in men. Physiology \& behavior 1989;45(3):639-47. [Google Scholar]

16. Wilk B, Bar-Or O. Effect of drink flavor and $\mathrm{NaCl}$ on voluntary drinking and hydration in boys exercising in the heat. Journal of Applied Physiology 1996;80(4):1112-7. [Google Scholar]

17. Zellner DA, Bartoli AM, Eckard R. Influence of color on odor identification and liking ratings. The American journal of psychology. 1991 Dec 1:547-61.

18. Musini VM, Wright JM. Factors affecting blood pressure variability: lessons learned from two systematic reviews of randomized controlled trials. PLoS One. 2009 May 22;4(5): e5673.

19. Suhr JA, Patterson SM, Austin AW, Heffner KL. The relation of hydration status to declarative memory and working memory in older adults. J Nutr Health Aging 2010;14(10):840-3. [Google Scholar]

20. Karsheva M, Dinkova P, Pentchev I, Ivanova T. Blood Rheology - A Key for Blood Circulation in The Human Body. Journal of The University of Chemical Technology and Metallurgy 2009;44(1):50 4. [Google Scholar] 